\title{
Design of the Compact Linear Collider main linac accelerating structure made from two halves
}

\author{
Hao Zha ${ }^{*}$ and Alexej Grudiev \\ CERN, European Organization for Nuclear Research, Geneva 1211, Switzerland
}

(Received 13 December 2016; published 14 April 2017)

\begin{abstract}
Milling on two longitudinally split halves is one method to manufacture accelerating structures. This method is simple and allows one to avoid electromagnetic fields at bonding joints, making it attractive in manufacturing high-gradient accelerating structures. An X-band structure design with strong wakefield damping based on this manufacturing approach is studied in this work as an alternative design for the Compact Linear Collider (CLIC) main linac accelerating structures. The geometry of the structure is optimized to greatly reduce the surface fields, improve the efficiency, and suppress the wakefield. This structure features the baseline design of the CLIC main linac with additional advantages. This study may serve as a reference for designing other high frequency-band corrugated structures.
\end{abstract}

DOI: 10.1103/PhysRevAccelBeams.20.042001

\section{INTRODUCTION}

High-gradient normal conducting accelerating structures are typically manufactured by precision turning or milling on separated disks [1]. These disks are brazed or bonded into a complete structure, as shown in Fig. 1. The disadvantage of this way of assembling the structure is the presence of a high magnetic field near the bonding joints, which may cause severe rf breakdown at high-gradient operation, as observed in experimental studies [2].

An alternative approach proposed in this work is to use precision milling to excavate half of the structure geometry on two metal blocks. Joining these two blocks composes a complete structure as shown in Fig. 2. The design of structure manufacturing in this way is also called an "open structure." Several studies have proposed designs of open structures, such as "muffin-tin structure" [3-5]. Manufacturing four quarter pieces of the complete structure geometry, known as the "quadrant structure" [6,7], was also studied.

Experimental evidence shows that metal surfaces that are in metal-to-metal contact but not bonded or brazed demonstrate poor performance during high-power operation $[9,10]$. Therefore, a finite gap is necessary in the open geometry design, as shown in Fig. 2. The gap geometry located outside cavities cuts off the accelerating mode so high power does not leak at the bonding place located at the side of the gap. This feature brings low requirements for

\footnotetext{
chah04@mails.tsinghua.edu.cn

Present address: Tsinghua University, Beijing, China.

Published by the American Physical Society under the terms of the Creative Commons Attribution 4.0 International license. Further distribution of this work must maintain attribution to the author(s) and the published article's title, journal citation, and DOI.
}

brazing and allows welding as an alternative joining technique.

Another potential advantage especially in the case of high frequency multicell structures is the greatly reduced number of parts to be assembled as well as reduced area of

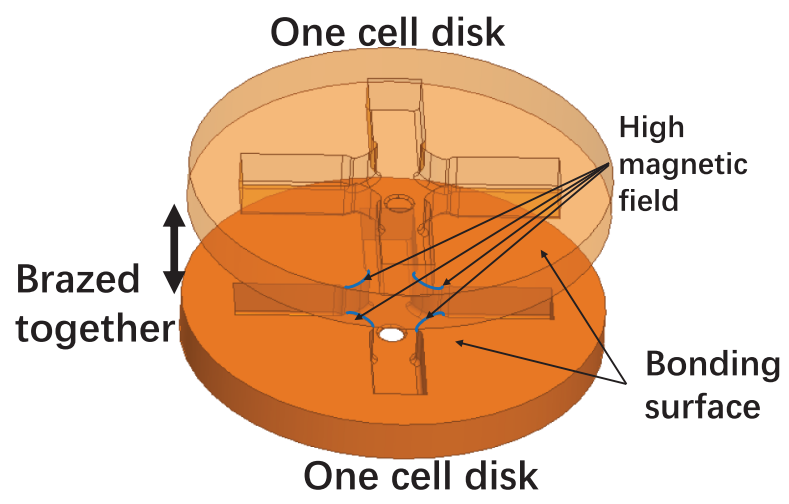

FIG. 1. Conventional way of manufacturing an accelerating structure.

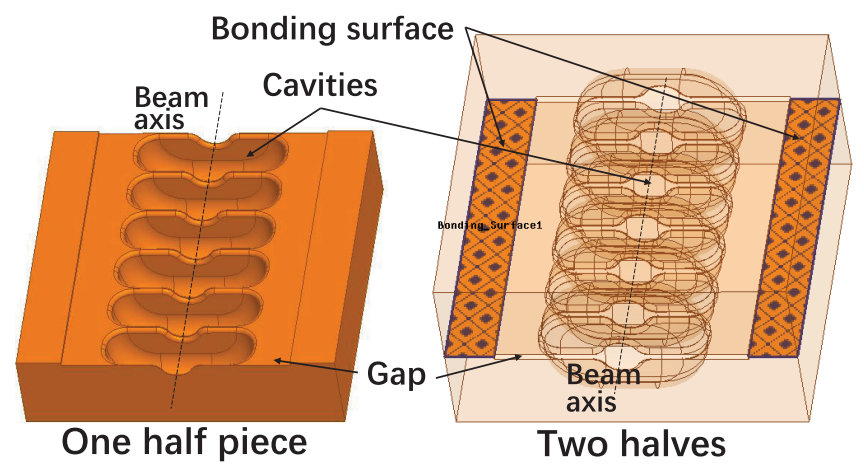

FIG. 2. Manufacturing accelerating structure by milling on two halves of copper plate (HFSS model [8]). 
the surface for bonding which is very demanding in terms of roughness and flatness. Both of these advantages promise great cost reduction in comparison to the standard assembly. Holding these benefits, the open structure is attractive for designs of high-frequency corrugate structures and has several useful applications [11-15].

The main linear accelerator (linac) of the Compact Linear Collider (CLIC) project $[16,17]$ uses an X-band waveguide damped structure as its baseline design [18]. The choke-mode structure [19] and the damped detuned structure [20] are also studied as alternative designs. All three structure designs use the conventional manufacturing approach and were designed and tested in recent years [21-28]. A design of the X-band strong damped open structure is studied in this work as the third alternative design of the CLIC main linac. Previous works realized the design and high power test of a prototype open structure without the full damping scheme [29]. The design is named CLIC-T24-Open, and its geometry conformed with that of CLIC-T24, thereby allowing comparison between the two manufacturing approaches. A structure prototype was manufactured at SLAC and was tested in the high power stand at CERN [30].

The beam dynamics of the CLIC main linac requires strong suppression to the long-range transverse wakefield in the structure design [31]. CLIC-T24-Open does not provide any suppression of high order modes (HOM). The structure geometry was modified to implement the strong damped design. New geometries of the iris and of the cavity wall were proposed to fit the required suppression of the wakefield and minimize the surface field (see Secs. II and III). This new geometry is useful for designing similar structures with a low surface field, which potentially leads to a high gradient. The strong damped design is suitable for other applications working in multibunch mode. The geometry of open structure has only two symmetric planes. As a result, a possible quadrupole component of the electromagnetic fields exists at the working frequency, which exerts a focusing or defocusing effect on the beam [32]. This quadrupole component and its effect have been observed in simulations and in experiments on other similar structures [14], respectively. Section IV discusses the study of the quadrupole field components.

\section{DESIGN OF STRUCTURE GEOMETRY}

\section{A. Basic concept}

The gap between two half metal blocks in the open structure design cuts off the accelerating mode. Some of the HOMs propagate in the gap and can be suppressed by rf damping loads placed in the gap. This mechanism of HOM suppression is similar to the mechanism in the slotted iris structure [32-34]. A study on CLIC beam dynamics reported that the beam-induced long-range transverse wakefield should be strongly suppressed by 2 orders of
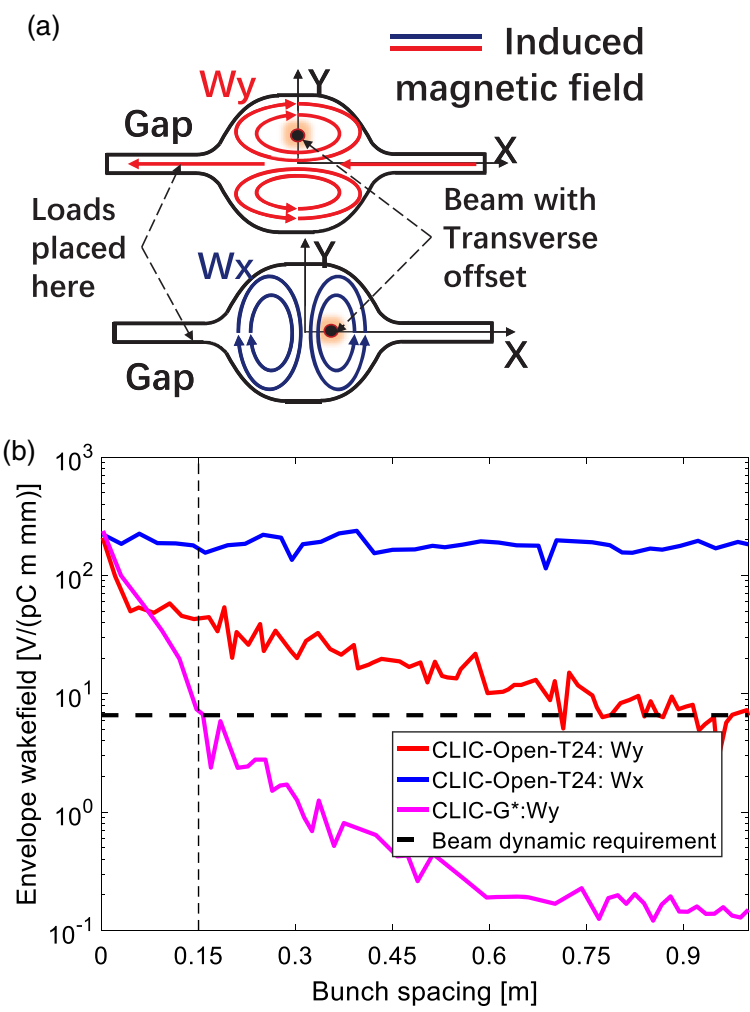

FIG. 3. Wakefield suppression of CLIC-T24-Open: (a) philosophy; (b) time-domain wakefield potential (CLIC-G* is the baseline main linac accelerating structure design).

magnitude within one bunch separation (0.5 ns) [31]. However, CLIC-T24-Open provides weak suppression to the transverse wakefield in only one polarization (defined as $W y$ ), as shown in Fig. 3. Thus, a geometric feature is implemented to provide strong suppression to both polarizations ( $W x$ and $W y$ ).

The new geometry design of the open structure is shown in Fig. 4. Several modifications of the geometry were introduced. Two waveguides are added on the side of the cavity to couple and damp the transverse wakefield similar to that in the baseline structure design of the CLIC main linac. The waveguides suppress HOMs in another

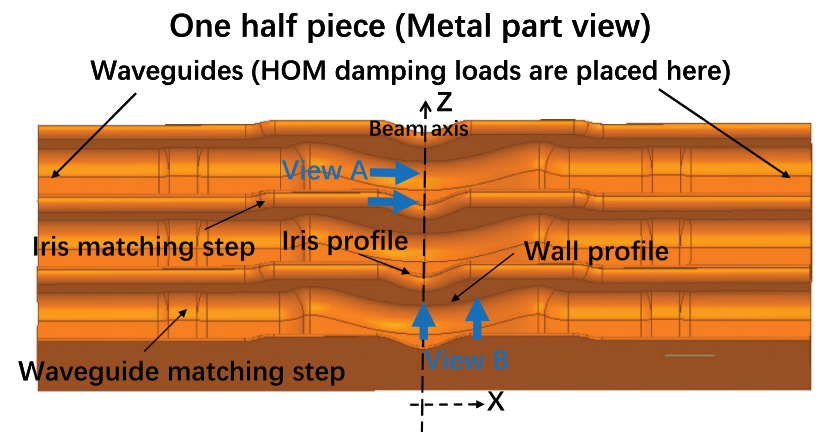

FIG. 4. New open geometry structure with full damping scheme. 


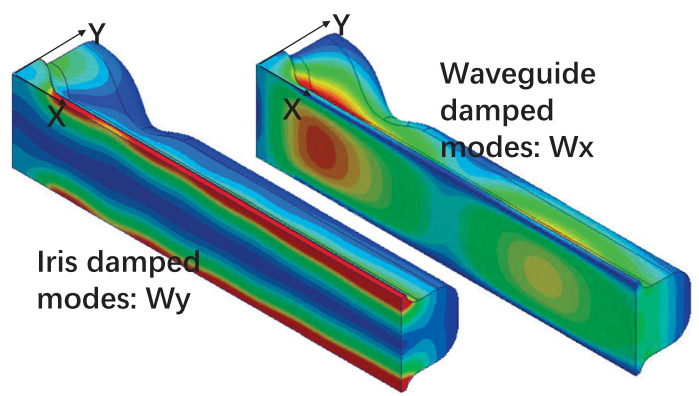

FIG. 5. Suppression of the HOMs in two polarizations.

polarization (Fig. 5). To minimize the surface field while maintaining the features of the accelerating mode in a reasonable level, new curves based on a cosinelike function are proposed for the profiles of the iris and the cell wall geometry (Fig. 6). The matching steps on the wall and iris are used to improve wakefield suppression.

\section{B. Optimization on surface fields}

In the design of the high-gradient accelerating structure, three quantities of surface fields are usually investigated: maximum electric field, maximum modified Poynting vector $S c$ [35], and maximum magnetic field. The maximum magnetic field is used to calculate the rise of the pulse surface heating temperature. The optimization of the magnetic field is related to the wall geometry, and the maximum electrical field and $S c$ are related to the iris geometry. Both of the geometries use cosinelike curves as

(a)
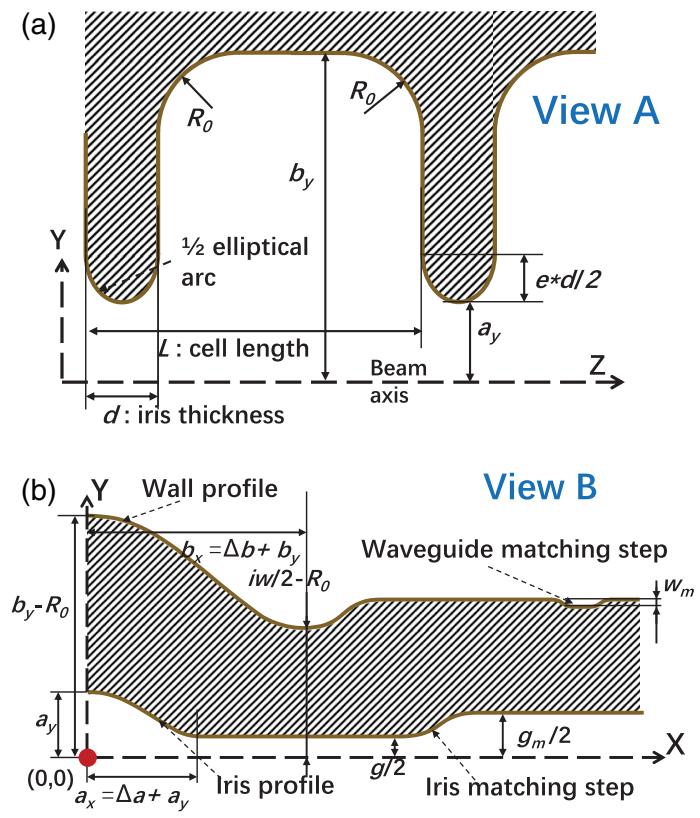

FIG. 6. Geometric parameters of the single cell: (a) Cut view of YZ plane; (b) view of the disk in the XY plane. See Fig. 4 for the orientation of each view. shown in Fig. 6. The functions of the curve in the wall profile are given in Eq. (1), and that of the iris profile is given in Eq. (2):

$$
\begin{aligned}
y= & \left(\frac{b_{y}}{2}-\frac{i w}{4}\right)\left\{\cos \left[\left(1-\beta+\frac{\beta x}{b_{x}}\right) \frac{\pi x}{b_{x}}\right]+1\right\} \\
& +\left(\frac{i w}{2}-R_{0}\right), \quad x \in\left(0, b_{x}\right),
\end{aligned}
$$

$$
y=\left(\frac{a_{y}}{2}-\frac{g}{4}\right)\left\{\cos \left[\left(1-\alpha+\frac{\alpha x}{a_{x}}\right) \frac{\pi x}{a_{x}}\right]+1\right\}+\frac{g}{2},
$$

$x \in\left(0, a_{x}\right)$,

Radio frequency simulations using HFSS code were carried out to study the surface fields of the first structure cell because in most cases it is the critical cell with highest surface field along the structure. Figure 7 shows the simulated results of the magnetic field distribution on the wall. To decrease the maximum magnetic field, the optimum parameters of the wall profile $\Delta b\left(\Delta b=b_{x}-b_{y}\right)$ and $\beta$ are $3.6 \mathrm{~mm}$ and 0.32 , respectively. The distributions of electrical field and $S c$ on the iris surface were calculated. The geometry of the iris profile also affects the group velocity in the cell, which determines the gradient of this cell for the given input power. Therefore, the group velocity should be kept constant when changing the iris geometry. Among all geometric parameters of the iris, $a_{y}$ and $\Delta a$ $\left(\Delta a=a_{x}-a_{y}\right)$ exert the most significant effect on the group velocity. Iris aperture $a_{y}$ is fixed and is equal to the one in the CLIC baseline design. $\Delta a$ is adjusted case by case in the simulation to make the comparisons under the same group velocity. Figure 8 shows the surface field for the iris profile variation of the first cell. $\alpha$ changes the field distribution of $S c$ but exerts a minimal effect on the maximum electrical field.

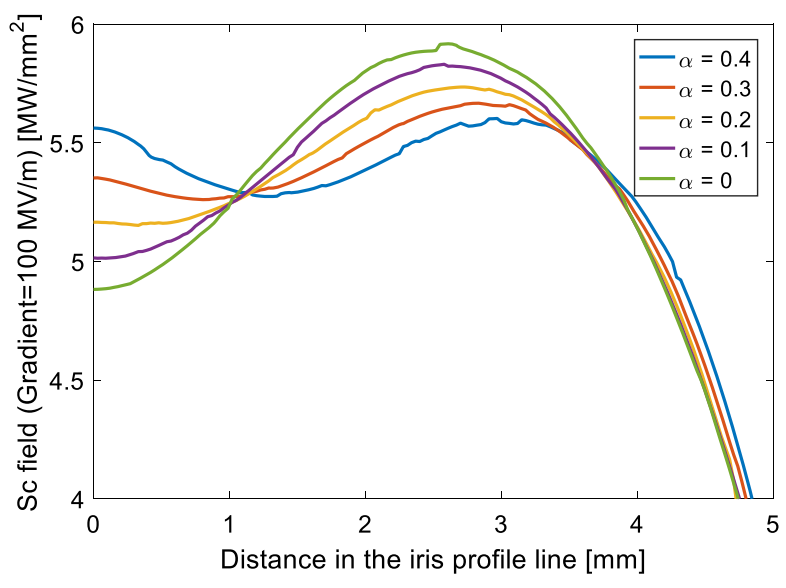

FIG. 7. Magnetic field distribution vs wall profile. 


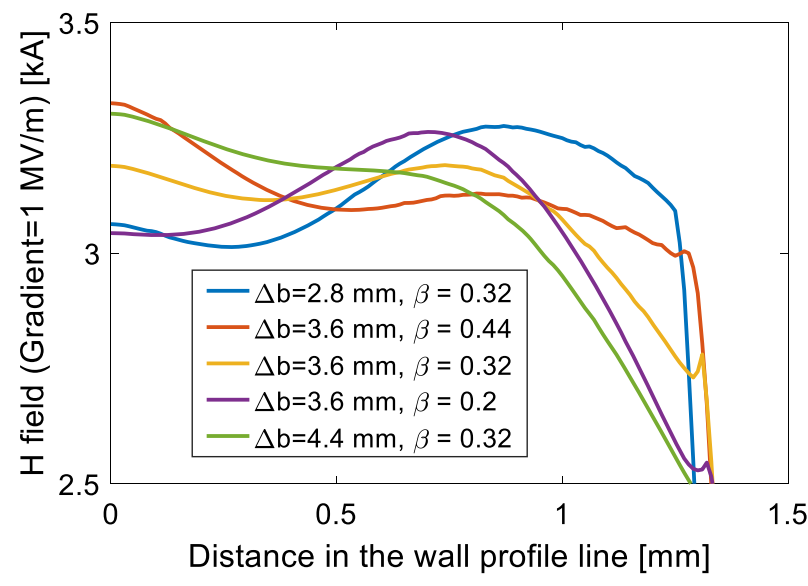

FIG. 8. $\quad S c$ vs wall profile.

\section{Optimization on the wakefield suppression}

The two waveguides on the side of the cell strongly damp the wakefield in the X polarization $(W x)$. However, the time-domain transverse wakefield simulations using GDFIDL code [36] show that the wakefield at the position of the second bunch $(\mathrm{s}=0.15 \mathrm{~m})$ is still too high (Fig. 9). The waveguides were connected to the prefect matching layers in the simulations. Analysis of the spectrum (or impedance) of the wakefield shows that the amplitude of the dipole mode near $16 \mathrm{GHz}$ dominates the wakefield value (Fig. 10). The suppression of this $16 \mathrm{GHz}$ mode can be improved by either increasing the waveguide opening and width or decreasing $\Delta b$. Larger waveguide dimensions not only increase the surface magnetic field but also reduce the efficiency (shunt impedance). Thus, we chose smaller $\Delta b(1.4 \mathrm{~mm})$ to improve the wakefield suppression and the shunt impedance. Although the magnetic field distribution is not optimum in this case, the maximum magnetic field is still lower than that of the CLIC baseline structure CLIC-G.

As shown in Fig. 10, a sharp peak appears near the $23 \mathrm{GHz}$ in the wakefield spectrum, which corresponds to a

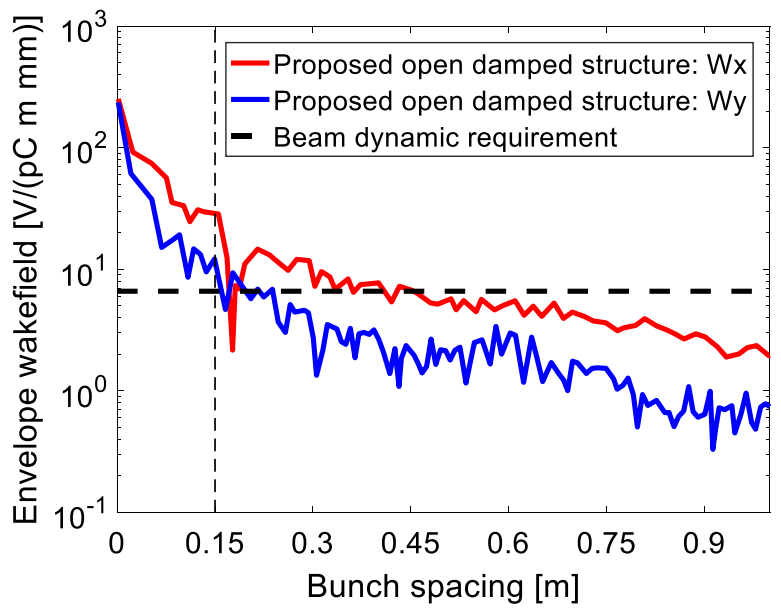

FIG. 9. Transverse wakefield of the proposed structure.

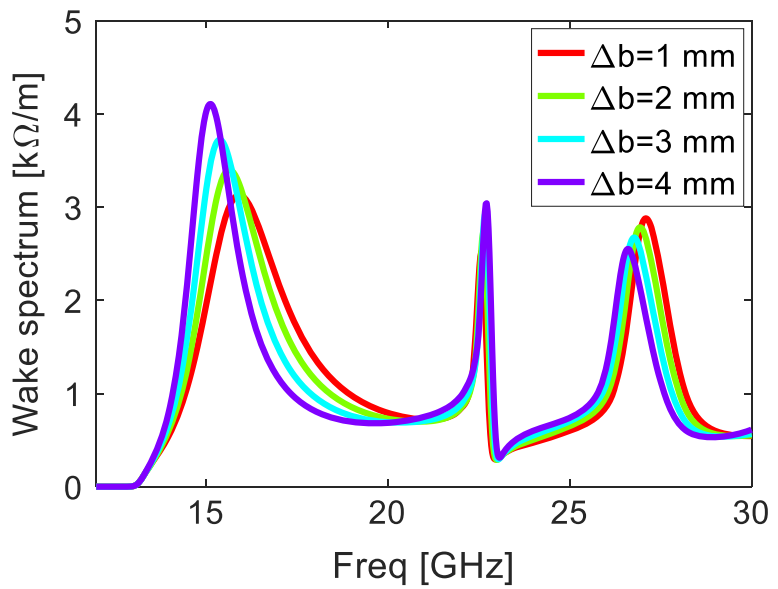

FIG. 10. Dependence of wakefield $(W x)$ on $\Delta b$.

poorly damped dipole mode. This mode significantly contributes to the wakefield at the position of all following bunches. The suppression to this dipole mode shows no significant dependence on the geometry of the wall profile, waveguide, or iris aperture. Investigation of all geometric
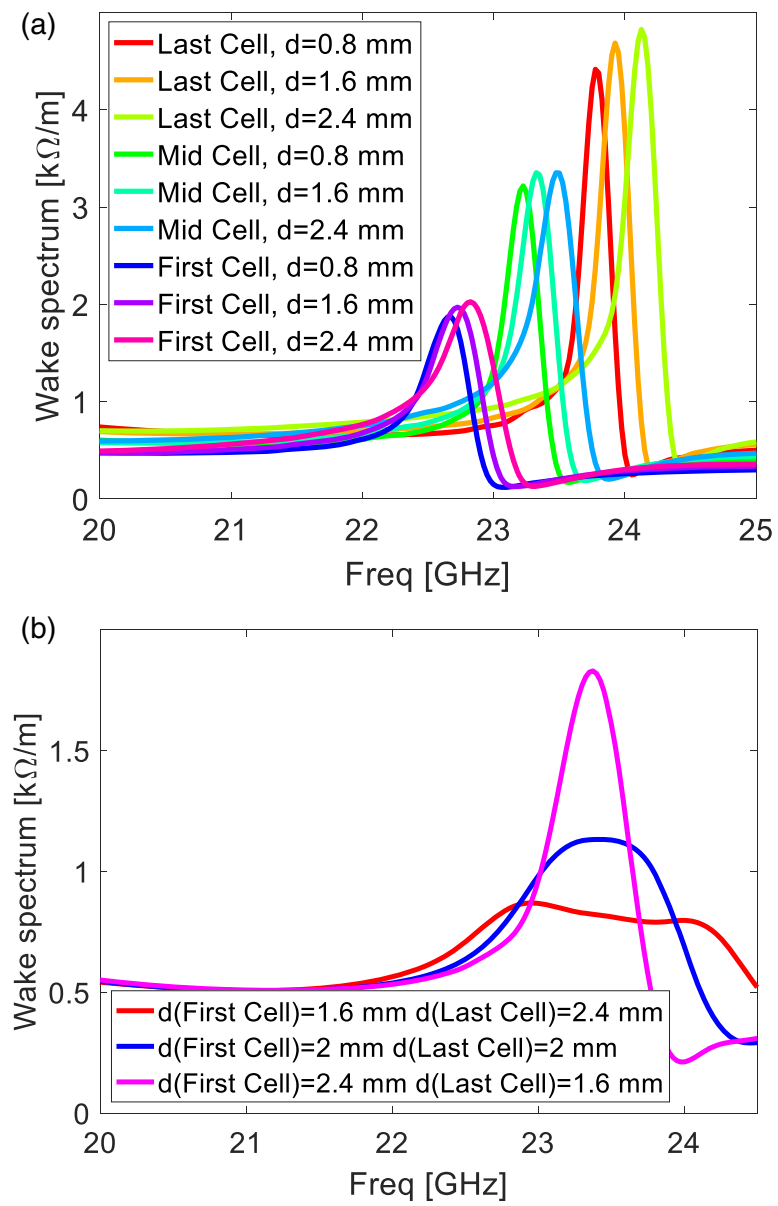

FIG. 11. Detuning the $23 \mathrm{GHz}$ mode ( $d$ is the iris thickness defined in Fig. 6): (a) Spectrum ( $W x$ ) of single cell; (b) spectrum $(W x)$ of the full structure. 

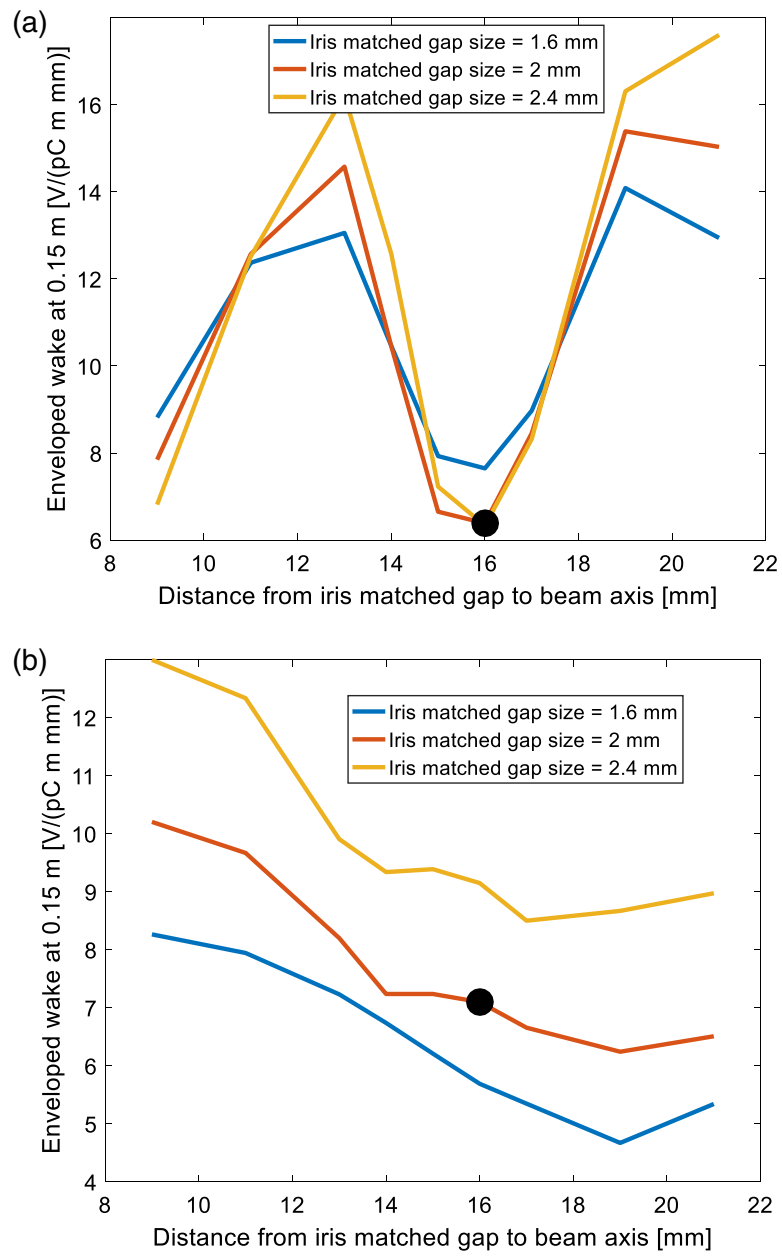

FIG. 12. Dependence of wakefield suppression on the iris matching step ( $g_{m}$ in Fig. 6): (a) $W x$; (b) $W y$. The solid point represents the optimum choice.

dimensions of the cell indicates that the frequency of this dipole mode could be changed by modifying the iris thickness. Therefore, the linear variation of the iris thickness among all the cells of one structure can detune this mode, which helps improve the overall wakefield suppression. Figure 11 shows the wakefield spectrum near $23 \mathrm{GHz}$ of the single cell and tapered structures. According to Fig. 11, it is preferred that the first cell in the tapered structure uses a thinner iris and the last cell uses a thicker one. Since the iris thickness has also an effect on the surface electrical or $S c$ field, a comprehensive study considering both wakefield suppression and surface field optimization was carried out. This study gives a solution that the iris thicknesses should be 1.6 and $2.3 \mathrm{~mm}$ for the first and last cells of the structure, respectively.

The wakefield in the $\mathrm{Y}$ polarization $(W y)$ is shown in Fig. 9. The iris matching step (Fig. 4) reflects part of the wakefield during propagation, and this reflection cancels the wakefield in the cavity. Figure 12 shows the enveloped amplitude of $W y$ near the position of the second bunch for

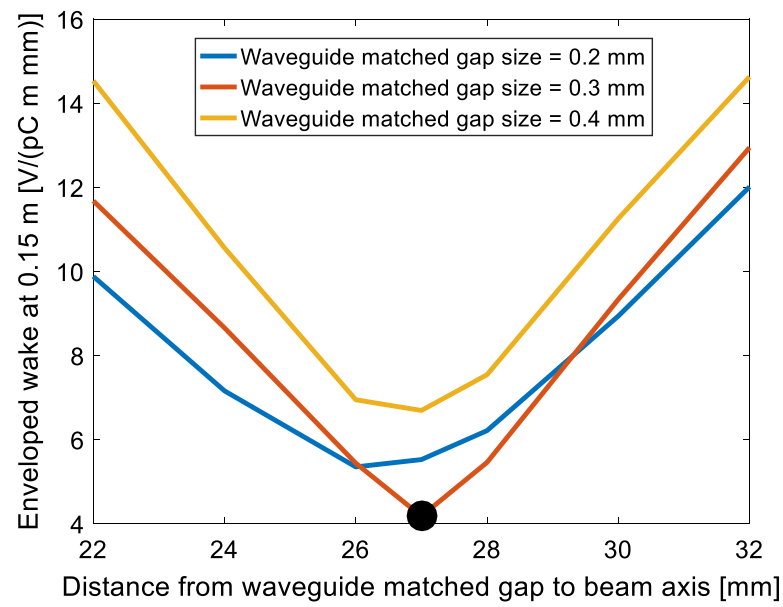

FIG. 13. Dependence of wakefield suppression $(W y)$ on the waveguide matching step ( $w_{m}$ in Fig. 6); the solid point represents the optimum choice.

different dimensions of iris matching steps. An optimum value is clearly present in the plot. The reflected wakefield enhances the amplitude at the position of the third bunch. However, the wakefield amplitude at this place is very small, and the overall wakefield suppression still improves.

As shown in Fig. 12, the iris matching step also changes the amplitude of $W x$, and the dimensions of the iris matching step for $W y$ are not optimal for $W x$. Hence, the waveguide matching step provides another free dimension to tune $W x$ and exerts no effect on $W y$. The enveloped amplitude of $W y$ near the position of the second bunch is plotted in Fig. 13

\section{DESIGN OF FULL TAPERED STRUCTURE}

\section{A. Design of coupler cell and full structure}

One structure unit of the baseline design for the CLIC main linac structure contains 26 regular cells and two compact coupler cells [18]. The radii of the iris aperture are 3.15 and $2.35 \mathrm{~mm}$ for the first and last structure cells, respectively. The compact coupler cells have a similar geometry to the regular cells but with two larger waveguides to transmit the power of the accelerating mode. This setup is also used to design the open damped structure, as shown in Fig. 14.

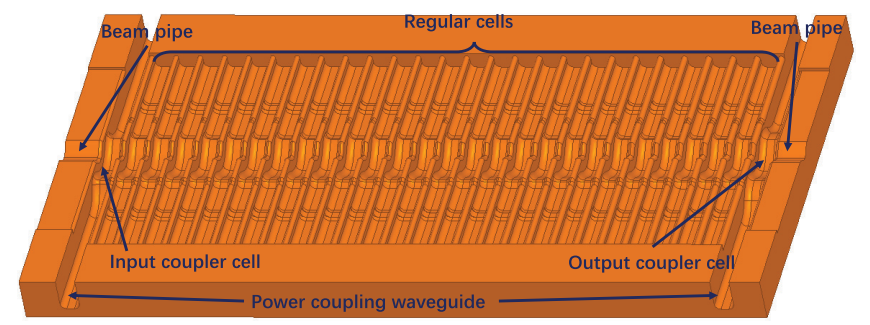

FIG. 14. Geometry of the coupler cell and the full tapered structure. Only one half is shown. 
TABLE I. The rf parameters of CLIC-HDS-A and CLIC-G* [18].

\begin{tabular}{lcc}
\hline \hline & CLIC-HDS-A & CLIC-G* \\
\hline Shunt impedance $[\mathrm{M} \Omega / \mathrm{m}]$ & 92.0 & 95.4 \\
Peak input power $[\mathrm{MW}]$ & 63.2 & 62.3 \\
rf to beam efficiency & $26.8 \%$ & $28.4 \%$ \\
Filling time [ns] & 69 & 66 \\
Maximum electric field $[\mathrm{MV} / \mathrm{m}]$ & 220 & 235 \\
Maximum $S c\left[\mathrm{MW} / \mathrm{mm}^{2}\right]$ & 5.13 & 5.54 \\
Maximum temperature rise $[\mathrm{K}]$ & 30 & 40 \\
\hline \hline
\end{tabular}

The rf parameters of the full tapered open damped structure are listed in Table I, together with those of the baseline design CLIC-G*. The field distributions on each structure cell are plotted in Fig. 15. Although the rf-tobeam efficiency of this open damped structure is slightly lower than that of CLIC-G*, the lower surface field and manufacturing method render the open damped structure a potentially better design. This structure design is named CLIC-HDS-A and will be manufactured and tested with high power in the near future.

\section{B. HOM damping loads design}

The HOM damping loads in the waveguide provide strong and broadband damping to the wakefield of two polarizations (Fig. 4) to meet the criteria of wakefield suppression. As shown in Fig. 16, the load adopts a smoothly tapered box geometry, and it sits on the middle

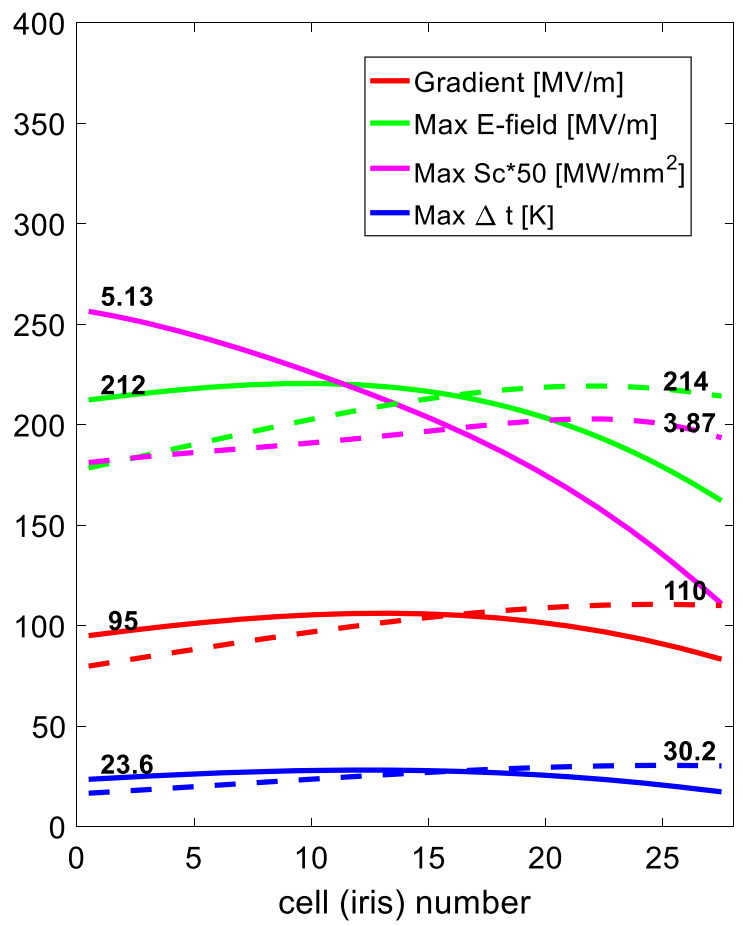

FIG. 15. Field distribution of structure cells (solid line: loaded $100 \mathrm{MV} / \mathrm{m}$; dashed line: unloaded $100 \mathrm{MV} / \mathrm{m}$ ).

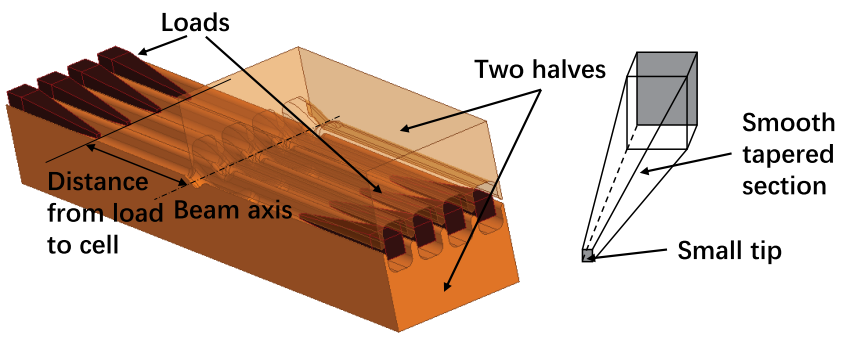

FIG. 16. Geometry of the HOM damping loads.

of two halves to absorb the propagating wakefield in both waveguides and the slot. The tip in the geometry of the load has small dimensions to reduce the reflection from the load. The long smooth tapered section in the geometry allows broadband absorption to the wakefield.

A silicon carbide-based material is used in the design of this damping load. This material is named Ekasic-P and is also used in the baseline CLIC-G* design (Fig. 17 of Ref. [18]). With the help of GDFIDL simulations on a full tapered structure of CLIC-HDS-A assembled with damping
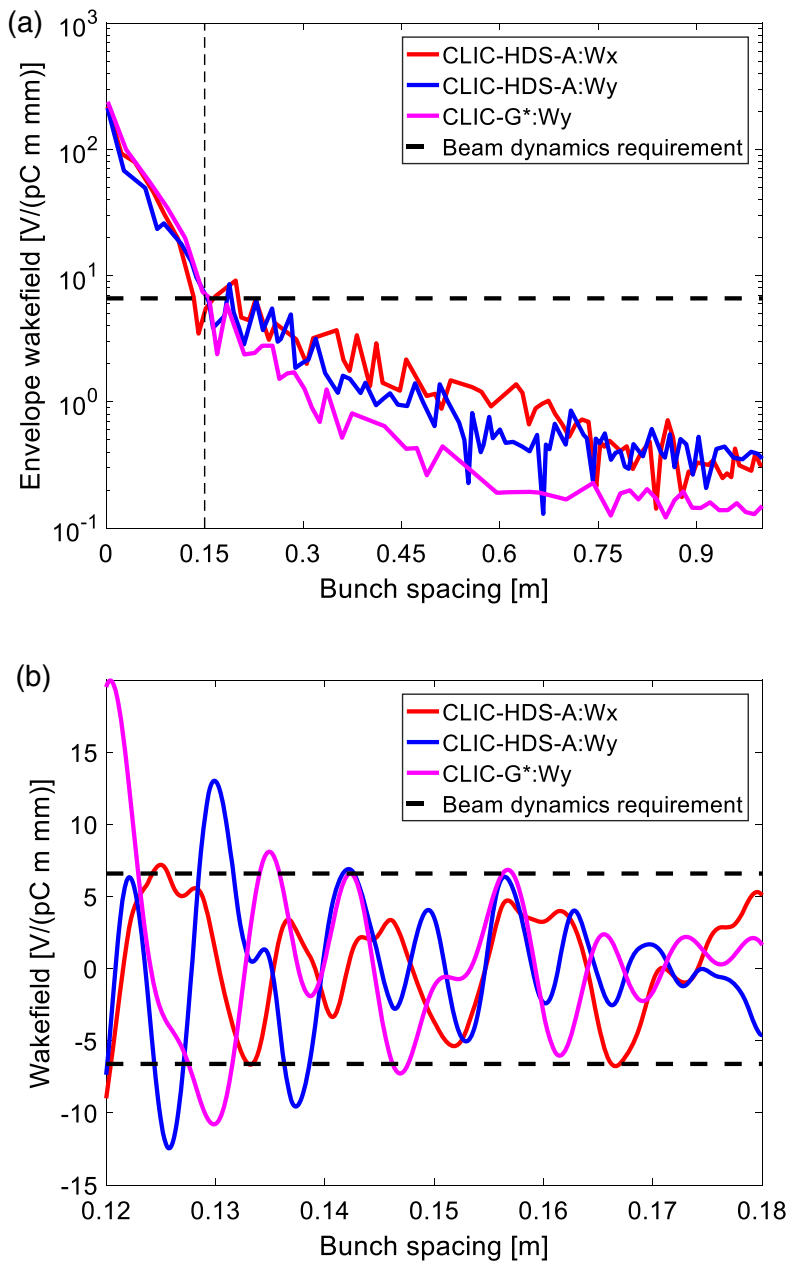

FIG. 17. Wakefield simulation results of CLIC-HDS-A: (a) Enveloped wake in log scale; (b) wake in linear scale. 
TABLE II. Transverse beam jitter amplification factors in wakefield suppression of CLIC-HDS-A.

\begin{tabular}{lccc}
\hline \hline Design & $F_{c}$ & $F_{\text {rms }}$ & $F_{\text {worst }}$ \\
\hline CLIC-G* $^{*}$ & 1.02 & 1.1 & 2.9 \\
$W x$ of CLIC-HDS-A & 1.03 & 2.0 & 5.2 \\
$W y$ of CLIC-HDS-A & 1.09 & 2.1 & 7.6 \\
Beam dynamics requirement & & $<5$ & $<20$ \\
\hline \hline
\end{tabular}

loads, the dimensions of this load were designed. The length of the designed load and the distance from the beam axis to the load are 3.7 and $4.3 \mathrm{~cm}$, respectively. The wakefield simulation results are plotted in Fig. 17 with two scales. Compared with the baseline CLIC-G* design, CLIC-HDS-A has a slightly weaker wakefield suppression on later than the third bunch. The overall wakefield effect on beam stability is stated by three factors, namely, $F_{c}$, $F_{\text {rms }}$, and $F_{\text {worst }}$. These factors present the amplification of transverse beam jitter in the main linac due to the longrange transverse wakefield in three ways. The $F_{c}$ is calculated based on that all bunches have same (coherent) transverse jitter, and $F_{\text {rms }}$ is calculated from the rms transverse jitter. $F_{\text {worst }}$ is calculated by the worst case. Further details of these factors are introduced in [31]. All factors are calculated using the wakefield at the positions of all 312 bunches in one full beam pulse and are listed in Table II. These factors for CLIC-HDS-A are slightly higher than those of the CLIC-G* but are still much lower than the level of beam stability requirement.

\section{HIGH-ORDER MULTIPOLAR FIELD COMPONENTS IN THE ACCELERATING MODE}

The geometry of the structure made from two halves has two planes of symmetry and brings quadruple field components to the accelerating mode. These quadruple field components exert transverse focusing or defocusing effect on the beams. As shown in Fig. 18, the phase of the maximum of the transverse quadrupolar kick has a $90^{\circ}$ shift with respect to the on-crest acceleration. Thus, if the bunch is accelerated in the on-crest phase, the focusing effect of the quadruple field components on the bunch center is zero.

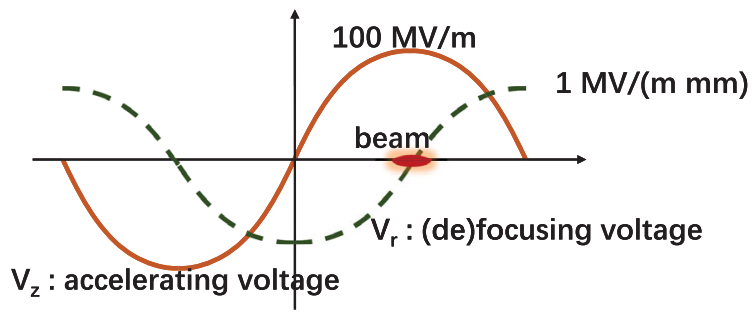

FIG. 18. Phase of quadruple field component and accelerating component.
As the bunch has a finite length, the other parts of the bunch will still experience the quadruple effect. This quadruple field component brings a time-varying focusing effect to the bunch and results in a beta function variation from the head to the tail of the bunch. This effect is similar to the Balakin-Novokhatsky-Smirnov (BNS) damping, which reduces the coherent transverse momentum of the bunch and improves the beam stability. The amplitude and effect of the rf quadrupole in the CLIC open damped structure are studied in this section.

Three-dimensional field simulations on the structure cell were carried out to gather field data and to calculate high-order multipolar components [37-41]. The transverse strength for the beam moving off-axis can be calculated from the field data by integrating Lorentz force equation [Eq. (3)]. The distribution of integrating transverse strength in the middle cell of CLIC HDS-A is plotted in Fig. 19. The strength of high-order field components can be calculated by angular Fourier transform on the off-axis transverse strength. The amplitudes of quadruple and octopole field components in the middle cell were calculated as $2.06 \mathrm{MV} / \mathrm{m} / \mathrm{mm}$ and $0.56 \mathrm{MV} / \mathrm{m} / \mathrm{mm}^{3}$ for a $100 \mathrm{MV} / \mathrm{m}$ gradient, respectively:
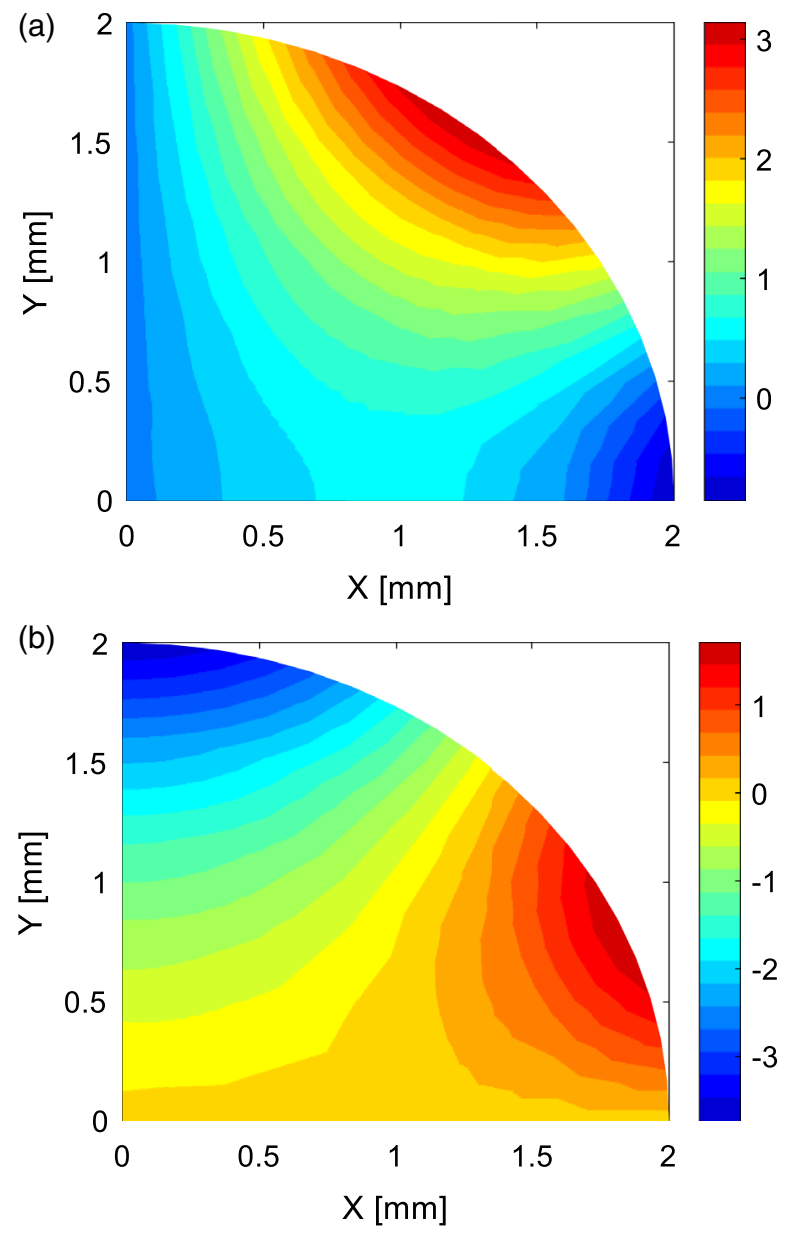

FIG. 19. Off-axis distribution of transverse deflection strength $[\mathrm{MV} / \mathrm{m}]$ to the beam: (a) $G_{x}$; (b) $G_{y}$. 


$$
\begin{aligned}
G_{x} & =\frac{1}{L} \int_{0}^{L}\left(E_{x}(x, y, z)-\frac{1}{c} B_{y}(x, y, z)\right) e^{j \frac{\omega z}{c}} d z \\
G_{y} & =\frac{1}{L} \int_{0}^{L}\left(E_{y}(x, y, z)+\frac{1}{c} B_{x}(x, y, z)\right) e^{j \frac{\omega z}{c}} d z,
\end{aligned}
$$

where $L$ is the structure length, and $\omega$ is the angular frequency. The electron beam is moving at the speed of light.

The calculated amplitudes of quadruple and octopole field components in the CLIC-HDS-A whole structure are $1.7 \mathrm{MV} / \mathrm{m} / \mathrm{mm}$ and $0.54 \mathrm{MV} / \mathrm{m} / \mathrm{mm}^{3}$ for a $100 \mathrm{MV} / \mathrm{m}$ average loaded gradient. The rms bunch length in the CLIC main linac is $44 \mu \mathrm{m}$. According to these numbers, the rms spread of focusing strength on the bunch is $18.8 \mathrm{kV} / \mathrm{m} / \mathrm{mm}$, while the corresponding rf quadruple gradient in magnetic units is $62.7 \mathrm{mT} / \mathrm{m}$. The gradient of quadruple magnets in the CLIC main linac is up to $200 \mathrm{~T} / \mathrm{m}$, and the average quadruple gradient of the full main linac is about $25 \mathrm{~T} / \mathrm{m}$. The quadruple field component results in about $0.25 \%$ variation (rms) in the quadruple strength. This number is comparable with the energy spread $(0.35 \%)$ of the beam in the main linac, which is used for BNS damping. Therefore, this time-depended quadruple strength could be used to provide the extra BNS damping to the beam and to reduce the requirement on the energy spread. However, this effect may also increase the projected emittance of the beam. A comprehensive research on the beam dynamics will be necessary to study the potential benefits or harm from the quadruple field components.

The high-order multipolar field components arise from asymmetric geometry. Consequently, their amplitude can change from different cavity geometries. Figures 20 and 21 show the plot of high-order multipolar field components

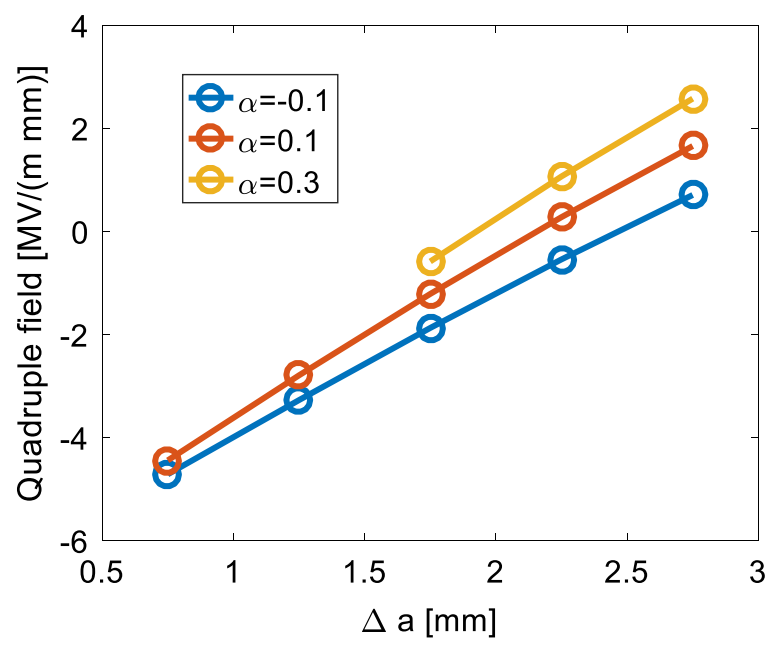

FIG. 20. Amplitude of quadruple components versus iris geometry.

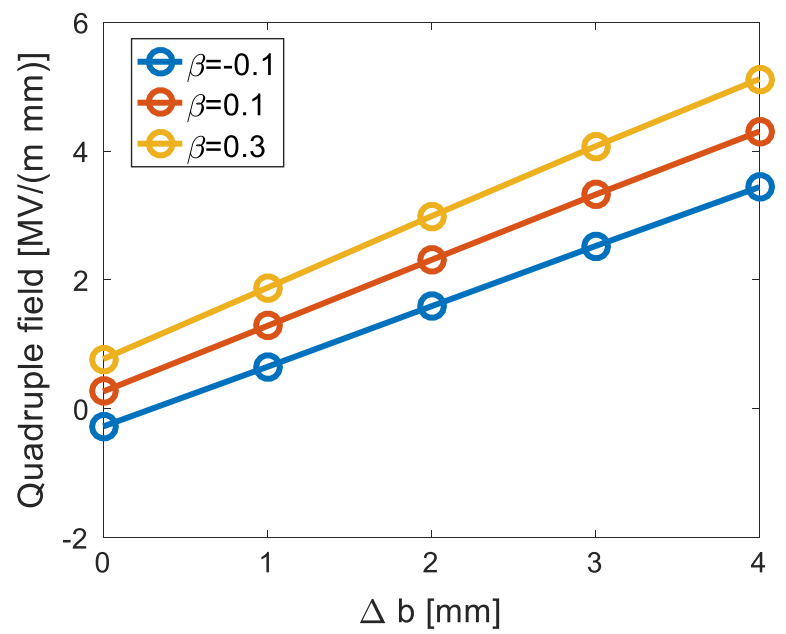

FIG. 21. Amplitude of quadruple components versus wall geometry.

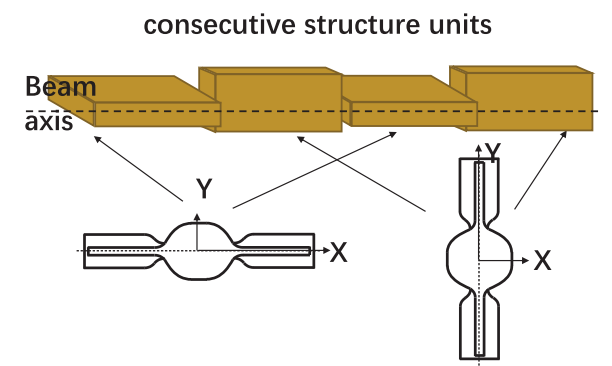

FIG. 22. Setup of rotating two consecutive structure units by 90 degrees.

versus different geometry dimensions. The enhancement or the elimination of the quadruple field components in the CLIC open damped structure could be achieved by properly designing the cell geometry. Another way to cancel the effect of quadruple field components to first order is through a $90^{\circ}$ rotation of two consecutive structure units as shown in Fig. 22. This setup not only cancels the quadruple field components but also reduces the deflection from long-range transverse wakefield because the beat of $W x$ and $W y$ is not the same as illustrated in Fig. 17.

\section{CONCLUSION}

An open damped accelerating structure is designed for the CLIC main linac. To implement the full wakefield damping scheme, two iris slots and two waveguides were introduced on the side of the cell for strong coupling of the dipolar transverse wake.

Several features in the geometry design were implemented for the open damped structure cell. A cosine function-based curve was proposed for the wall and iris profile to reduce the surface fields. Iris thickness varies from cell to cell to detune the dipole mode and to improve the suppression to the wakefield. Matching steps on the iris 
slot and waveguides reduce the wakefield at the position of the second bunch.

A tapered structure with compact coupler cells and HOM damping loads has been designed. The structure is named "CLIC-HDS-A" and will be built and tested in the near future. Compared with the baseline design of the CLIC main linac (CLIC-G*), CLIC-HDS-A has a lower surface field and a slightly lower rf-to-beam efficiency. Simulations using GDFIDL code show that the CLIC-HDS-A design meets the criteria of wakefield suppression required by CLIC beam dynamics.

A significant quadruple field component exists in the accelerating mode of the open damped structure because of its two-plane symmetry. The amplitude of this quadruple field component depends on the geometric dimensions. This quadruple field component brings time-varying focusing-defocusing strength and results in the beta function spread along the bunch, which is useful for BNS damping. Additional studies on beam dynamics considering this quadruple field component effect are necessary.

\section{ACKNOWLEDGMENTS}

The authors thank Valery Dolgashev, Walter Wuensch, Daniel Schulte, and Andrea Latina for the fruitful discussions.

[1] J. Wang et al., Fabrication technologies of the high gradient accelerator structures at $100 \mathrm{MV} / \mathrm{m}$ range, in Proceedings of the International Particle Accelerator Conference, Kyoto, Japan (ICR, Kyoto, 2010).

[2] Ana Teresa Perez Fontenla, TD24 post-mortem SEM analysis, at the CLIC rf development meeting (2014), https://indico.cern.ch/event/320252/.

[3] W. Weingarten and R. Blaschke, Electromagnetic fields in a muffin tin cavity, Nucl. Instrum. Methods 156, 597 (1978).

[4] P. Chou, G. Bowden, M. Copeland, H. Henke, A. Menegat, and R. Siemann, Design and fabrication of a traveling wave muffin-tin accelerating structure at $90 \mathrm{GHz}$, in Proceedings of the Particle Accelerator Conference, Vancouver, BC, Canada, 1997 (IEEE, New York, 1997), p. 464.

[5] A. Blednykh and H. Henke, A HOM damped planar accelerating structure, in Proceedings of 9th European Particle Accelerator Conference, EPAC-2004 (Jacow, Lucerne, Switzerland, 2004), pp. 982-984.

[6] T. Higo et al., Fabrication of a quadrant-type accelerator structure for CLIC, in Proceedings of 9th European Particle Accelerator Conference, EPAC-2004 (Jacow, Genoa, Italy, 2008), pp. 2716-2718.

[7] T. Abe, Basic study on high-gradient accelerating structures at KEK/Nextef, at The Eighth Workshop on High-Gradient Acceleration, HG2015 (Beijing, China, 2015), https://indico.cern.ch/event/358352/contributions/ 1770613/.

[8] HFSS, www.ansys.com.
[9] L. Laurent, S. Tantawi, V. Dolgashev, C. Nantista, Y. Higashi, M. Aicheler, S. Heikkinen, and W. Wuensch, Experimental study of rf pulsed heating, Phys. Rev. ST Accel. Beams 14, 041001 (2011).

[10] Z. Peng, Effects of surface roughness on electrical contact, rf heating and field enhancement, Doctoral dissertation, The University of Michigan, 2012.

[11] M. Dal Forno, V. Dolgashev, G. Bowden, C. Clarke, M. Hogan, D. McCormick, A. Novokhatski, B. O'Shea, B. Spataro, S. Weathersby, and S. G. Tantawi, Rf breakdown measurements in electron beam driven $200 \mathrm{GHz}$ copper, and copper-silver accelerating structures, Phys. Rev. Accel. Beams 19, 111301 (2016).

[12] M. Dal Forno, V. Dolgashev, G. Bowden, C. Clarke, M. Hogan, D. McCormick, A. Novokhatski, B. Spataro, S. Weathersby, and S. G. Tantawi, Experimental measurements of rf breakdowns, and deflecting gradients in mmwave metallic accelerating structures, Phys. Rev. Accel. Beams 19, 051302 (2016).

[13] M. Dal Forno, V. Dolgashev, G. Bowden, C. Clarke, M. Hogan, D. McCormick, A. Novokhatski, B. Spataro, S. Weathersby, and S. G. Tantawi, Rf breakdown tests of mmwave metallic accelerating structures, Phys. Rev. Accel. Beams 19, 011301 (2016).

[14] D. Wang, S. Antipov, C. Jing, J. G. Power, M. Conde, E. Wisniewski, W. Liu, J. Qiu, G. Ha, V. Dolgashev, C. Tang, and W. Gai, Interaction of an Ultrarelativistic Electron Bunch Train with a W-Band Accelerating Structure: High Power and High Gradient, Phys. Rev. Lett. 116, 054801 (2016).

[15] A. Nassiri, R. L. Kustom, F. E. Mills, Y. W. Kang, A. D. Feinermant, P. J. Matthews, T. L. Willket, D. Grudzien, J. Song, and D. Horan, A 50-MeV mm-wave electron linear accelerator system for production of tunable short wavelength synchrotron radiation, in Proceedings of IEEE International Electron Devices Meeting, IDEM-93 (IEEE, Washington, DC, USA, 1993), pp. 169-172.

[16] R. Tomás, Overview of the Compact Linear Collider, Phys. Rev. ST Accel. Beams 13, 014801 (2010).

[17] CLIC Conceptual Design Report (CDR), CERN-2012-007 (2012), http://project-clic-cdr.web.cern.ch/projectCLICCDR/CDR_Volume1.pdf.

[18] H. Zha and A. Grudiev, Design, and optimization of Compact Linear Collider main linac accelerating structure, Phys. Rev. Accel. Beams 19, 111003 (2016).

[19] H. Zha, J. Shi, H. Chen, A. Grudiev, W. Wuensch, C. Tang, and W. Huang, Choke-mode damped structure design of the Compact Linear Collider main linac, Phys. Rev. ST Accel. Beams 15, 122003 (2012).

[20] V. F. Khan, A. D’Elia, R. M. Jones, A. Grudiev, W. Wuensch, G. Riddone, and V. Soldatov, Wakefield and surface electromagnetic field optimization of manifold damped accelerating structures for CLIC, Nucl. Instrum. Methods Phys. Res., Sect. A 657, 131 (2011).

[21] T. Higo et al., Advances in X-band TW accelerator structures operating in the $100 \mathrm{MV}=\mathrm{m}$ regime, in Proceedings of the International Particle Accelerator Conference, Kyoto, Japan (ICR, Kyoto, 2010), p. 3702.

[22] T. Higo, Progress of X-band accelerating structures, in Proceedings of the 25th International Linear Accelerator 
Conference, LINAC-2010, Tsukuba, Japan (KEK, Tsukuba, Japan. 2010).

[23] A. Degiovanni, W. Wuensch, and J. G. Navarro, Comparison of the conditioning of high gradient accelerating structures, Phys. Rev. Accel. Beams 19, 032001 (2016).

[24] H. Zha, A. Latina, A. Grudiev, G. De Michele, A. Solodko, W. Wuensch, D. Schulte, E. Adli, N. Lipkowitz, and G. S. Yocky, Beam-based measurements of long range transverse wakefields in the CLIC main linac accelerating structure, Phys. Rev. Accel. Beams 19, 011001 (2016).

[25] X. Wu et al., High power test of X-band single cell HOMfree choke-mode damped accelerating structure made by Tsinghua University, in Proceedings of the Seventh International Particle Accelerator Conference, IPAC2016 (Jacow, Busan, Korea, 2016), pp. 3881-3883.

[26] F. Wang, C. Adolphsen, and C. Nantista, Performance limiting effects in $\mathrm{X}$-band accelerators, Phys. Rev. ST Accel. Beams 14, 010401 (2011).

[27] R. M. Jones, Wakefield suppression in high gradient linacs for lepton linear colliders, Phys. Rev. ST Accel. Beams 12, 104801 (2009).

[28] J. Shi, A. Grudiev, and W. Wuensch, Tuning of X-band traveling-wave accelerating structures, Nucl. Instrum. Methods Phys. Res., Sect. A 704, 14 (2013).

[29] H. Zha et al., Rf design of the CLIC structure prototype optimized for manufacturing from two halves, in Proceedings of the Sixth International Particle Accelerator Conference, IPAC-2015, (Jacow, Richmond VA, USA, 2015), pp. 2147-2149.

[30] N. C. Lasheras et al., Fabrication and high-gradient testing of an accelerating structure made from milled halves, in Proceeding of 28th Linear Accelerator Conference, LINAC-16 (Jacow, Michigan, USA, 2016), pp. 226-228.

[31] D. Schulte, Multibunch calculations in the CLIC main linac, in Proceedings of the 23rd Particle Accelerator Conference, Vancouver, Canada, 2009 (IEEE, Piscataway, NJ, 2009).

[32] A. Latina, A. Grudiev, and D. Schulte, Study of fundamental mode multipolar kicks in double and single-feed power couplers for the CLIC main linac accelerating structure, in Proceedings of the 3rd International Particle Accelerator Conference, New Orleans, LA, 2012 (IEEE, Piscataway, NJ, 2012), WEPPR067.

[33] S. Dobert et al., High power test of an X-band slotted-iris accelerator structure at NLCTA, Report No. SLAC-PUB12788.

[34] E. Jensen, I. Syratchev, and W. Wuensch, Slottediris structure studies, in Proceedings of the 2001 Particle Accelerator Conference, PAC-01 (Jacow, Chicago, USA, 2001), pp. 855-857.

[35] A. Grudiev, S. Calatroni, and W. Wuensch, New local field quantity describing the high gradient limit of accelerating structures, Phys. Rev. ST Accel. Beams 12, 102001 (2009).

[36] Gdfidl, www.gdfidl.de.

[37] J. Barranco García, R. De Maria, A. Grudiev, R. Tomás García, R. B. Appleby, and D. R. Brett, Long term dynamics of the high luminosity Large Hadron Collider with crab cavities, Phys. Rev. Accel. Beams 19, 101003 (2016).

[38] P. K. Ambattu, G. Burt, A. Grudiev, V. Dolgashev, and A. Dexter, Coupler induced monopole component and its minimization in deflecting cavities, Phys. Rev. ST Accel. Beams 16, 062001 (2013).

[39] Y. Huang, H. Wang, R. A. Rimmer, S. Wang, and J. Guo, Ultrafast harmonic rf kicker design, and beam dynamics analysis for an energy recovery linac based electron circulator cooler ring, Phys. Rev. Accel. Beams 19, 084201 (2016).

[40] B. Xiao, L. Alberty, S. Belomestnykh, I. Ben-Zvi, R. Calaga, C. Cullen, O. Capatina, L. Hammons, Z. Li, C. Marques, J. Skaritka, S. Verdú-Andres, and Q. Wu, Design, prototyping, and testing of a compact superconducting double quarter wave crab cavity, Phys. Rev. ST Accel. Beams 18, 041004 (2015).

[41] J. Barranco García, R. Calaga, R. De Maria, M. Giovannozzi, A. Grudiev, and R. Tomás, Study of multipolar rf kicks from the main deflecting mode in compact crab cavities for LHC, in Proceedings of the 3rd International Particle Accelerator Conference, New Orleans, LA, 2012 (Ref. [33]), TUPPR027. 